\title{
Neurocognitive flexibility, perfectionism, obsessive beliefs in patients with obsessive compulsive disorder
}

\author{
Obsesif kompulsif bozuklukta nörobilişsel esneklik, mükemmelliyetçilik ve \\ obsesif inanışlar
}

Betül Önder Uzgan1, Melike Tetik Oktay², Cansu Aykaç², Çağatay Ermiş3, Tunç Alkın4
${ }^{1}$ Res. Assis., Çanakkale Onsekiz Mart University, Faculty of Arts And Science, Department of Biology, Çanakkale, Turkey
https://orcid.org/0000-0002-6423-6704
2Res. Assis., Dokuz Eylul University, Institute of Health And Science, Department of Neurosciences, İzmir, Turkey
https://orcid.org/0000-0002-0367-3820-https://orcid.org/0000-0001-8702-5478
${ }^{3}$ M.D., Dokuz Eylul University Faculty Of Medicine, Department of Child and Adolescent Psychiatry, İzmir, Turkey
https://orcid.org/0000-0002-8412-8049
4Prof., Dokuz Eylul University Faculty Of Medicine, Department of Psychiatry, İzmir, Turkey https://orcid.org/0000-0002-1472-7433

SUMMARY

Objective: Obsessive Compulsive Disorder (OCD) is a heteregenous psychiatric disorder. In this study, three possible etiopathogenic factors, neurocognitive flexibility, perfectionism, and obsessive beliefs in patients with $\mathrm{OCD}$, were evaluated and compared with healthy controls. The hypothesis is neurocognitive flexibility, obsessive beliefs, and perfectionism may have a role in the formation of OCD symptoms. Furthermore, as perfectionism and obsessive beliefs increase, neurocognitive flexibility may deteriorate further. Method: The study included 66 OCD patients and 75 healthy controls with no psychiatric history. Berg Card Sorting Test (BCST), Trail Making Test (TMT) and Category Fluency (CF) Test were used to assess neurocognitive flexibility; Hewitt Multidimensional Perfectionism Scale (HMPS) and Obsessional Beliefs Questionnaire-44 (OBQ-44) were administered to evaluate perfectionism and obsessive beliefs of participants. Structured Clinical Interview for DSM-IV (SCID-I) was administered to participants. YaleBrown Obsession Compulsion Scale(Y-BOCS) was applied to evaluate severity of obsessions/compulsions, while Hamilton Depression Rating Scale (HAM-D) was used to evaluate the severity of depression of patients. Results: Patients had high level perfectionist personality traits, and their levels of obsessive beliefs were higher than the healthy group. Trail Making Test performance was poorer in patients with $O C D$. There was no significant relationship between obsessive beliefs, perfectionism and neurocognitive flexibility. However, these variables differed among OCD-subtypes. Discussion: Further studies may investigate various OCD-subtypes by diversifying cognitive flexibility measurement along with biological variables.

Key Words: Cognition, obsessive-compulsive disorder, perfectionism

(Turkish J Clinical Psychiatry 2021;24:439-449)

DOI:10.5505/kpd.2021.90187

\section{ÖZET}

Amaç: Obsesif Kompulsif Bozukluk (OKB) heterojen bir ruhsal bozukluktur. Bu çalışmada OKB oluşumundan sorumlu olabileceği düşünülen nörobilişsel esneklik, mükemmelliyetçilik ve obsesif inanışlar arasındaki ilişki sağlıklı kontroller ile karşılaştırılarak değerlendirildi. Çalışma hipotezi nörobilişsel esneklik, mükemmelliyetçilik ve obsesif inançların OKB belirtilerinin oluşumunda rol oynayabileceği ve bu inançlar yoğunlaştıkça nörobilişsel esnekliğin daha çok bozulabileceğidir. Yöntem: 66 OKB tanılı ve hiçbir psikiyatrik öyküsü olmayan 75 sağlıklı birey çalışmaya katıldı. Katılımcıların, Berg Kart Eşleme Test (BKET), İz Sürme Testi (IST) ve Kategori Akıcılık (KA) Testiyle nörobilişsel esneklik, Hewitt Çok Boyutlu Mükemmelliyetçilik Ölçeği (HÇBMÖ) ve Obsesif İnanışlar Ölçeği-44 (OiÖ-44) ile mükemmelliyetçilik ve obsesif inançlarının düzeyleri değerlendirildi. Katılımcılara DSMIV Eksen I Bozuklukları için Yapılandırılmış Klinik Görüşme (SCID-I) uygulandı. Hastaların, Yale-Brown Obsesyon Kompulsiyon Ölçeğiyle (Y-BOCS) obsesyonlar ve kompulsiyonlar, Hamilton Depresyon Derecelendirme Ölçeğiyle (HAM-D) depresyon şiddeti değerlendirildi. Bulgular: Hastaların, sağlıklı gruba göre mükemmelliyetçi özellikleri ve obsesif inanışları daha yüksekti. Hastaların İz Sürme Testi (IST) performansları kontrollere göre daha kötüydü. Mükemmelliyetçilik ve obsesif inanç düzeyleri ile nörobilişsel esneklik arasında anlamlı ilişki bulunamamıştır. Fakat OKB alt-grupları arasında bu değişkenlerde farklılıklar vardı. Sonuç: İleride, biyolojik değişkenlerle birlikte bilişsel esneklik ölçüm araçları çeşitlendirilerek daha fazla OKB alt-grupları değerlendirilebilir.

Anahtar Sözcükler: Kognisyon, mükemmelliyetçilik, obsesif-kompulsif bozukluk 


\section{INTRODUCTION}

Neurocognitive studies are promising in understanding the formation and clinical expression of the symptoms of OCD (Obsessive Compulsive Disorder). Executive functions refer to top-down mental processes that comprise concentration, attention, planning, formulating goals $(1,2)$. Previous studies showed that patients with OCD suffered from executive dysfunctions (3-6). Cognitive flexibility is one of the possible executive functions that may be related to OCD. It is defined as an ability to react adaptively and reconstruct knowledge in the presence of changing situations $(7,8)$. Researchers report that the performance of OCD patients in those neurocognitive tests that assess frontal lobe activity is poorer than healthy controls, which reflects cognitive flexibility disruptions in OCD $(9,10)$. The disruption of flexibility could be related to the severity of clinical symptoms in OCD and the course of the disorder (11). Given that a high level of obsessive beliefs in OCD may result in decreased performance on Wisconsin Card Sorting Test (WCST), it could be concluded that strong obsessive beliefs may contribute to the problems of cognitive flexibility in patients with OCD. Also, the poorer performance of OCD patients in Trail Making Test (TMT) trials than healthy controls point out the inability to changing strategy and inhibition (9). Consequently, patients may have difficulty in shifting from obsessional thoughts (12). Recent evidence revealed that OCD patients aged between 7-17 showed not only cognitive inflexibility but also problems in other neurocognitive abilities such as planning and visual processing (13).

OCD patients may show different clinical features that constitute OCD subtypes. In general, these subtypes include symmetry/ordering, forbidden thoughts, contamination/cleaning, and hoarding obsessions/compulsions (14-16). Several attempts have been made to investigate differences in neuropsychological performance among subtypes of OCD. For example, symmetry/ordering subtype of OCD have more difficulty in attention, visuospatial ability, verbal working memory, and cognitive flexibility than obsession/control subtype of OCD, and the symptom severity may be related to decreased cognitive flexibility in symmetry/orde- ring subtype compared to obsession/control subtype (17).

Some of the brain regions show distinct activations based on OCD subtypes; for example, contamination subtypes are related to limbic regions' dysfunctions, whereas basal ganglion dysfunctions link to checking/control subtypes of OCD (18). Furthermore, activation of ventromedial prefrontal regions and caudate nucleus in contamination/washing subtype and activation of regions responsible for motor functions and attention (putamen, thalamus) in checking subtype were higher than healthy subjects. These findings indicate OCD is a heterogeneous disorder, and neurobiological factors for each OCD subtype may differ (19).

In the cognitive model of OCD, the dysfunctional beliefs (perfectionism, intolerance of uncertainty, inflated responsibility, overestimation of threat and overimportance of thoughts, and importance of controlling one's thoughts) are thought as possible reasons lie behind OCD (20).

Assuming that impairments in neurocognitive functions and dysfunctional obsessive beliefs lie beneath the symptoms seen in OCD, it may constitute a link between the clinical expression and neurobiological etiology of this disorder. Therefore, we aimed to evaluate the possible relationship among dysfunctional obsessive beliefs, perfectionism, and neurocognitive inflexibility to comprehend the association between clinical symptoms and the neurobiological etiology of OCD. We hypothesized that OCD patients with a high level of obsessive beliefs and perfectionism would also show greater neurocognitive inflexibility. The current research aimed to contribute to this growing area of research by exploring the neurocognitive etiology of OCD and its clinical signs.

\section{METHODS}

\section{Participants}

One hundred forty-one participants (66 OCD patients and 75 healthy controls (HC) matched for sex, age, and education level) were included in the 
study. Patients were recruited from Ege University Faculty of Medicine Psychiatry clinics by applying a simple random sampling method. Inclusion criteria were as follows: i)aged between $18-60$, ii) ability to provide informed consent, iii) at least having primary school degree, iv) diagnosed with OCD (patient group), v) having no psychiatric conditions based on Structural Clinical Interview for Axis I Disorders (SCID-I) (control group) (21), Exclusion criteria were as follows: Diagnosed with any psychiatric condition such as schizophrenia, bipolar disorder or psychotic disorders, presence of neurological disorders, recent alcohol and substance use disorders, and mental retardation based on interviews using SCID-1 and receiving electroconvulsive therapy. Participants who have any psychiatric conditions were excluded from the study. OCD patients who have comorbid psychiatric disorders mentioned above were excluded from the study. The study was approved by Ege University Ethics committee. All participants gave their informed consent before participation. Some of the patients were excluded due to having comorbid psychiatric disorders (schizophrenia and alcohol dependency, respectively), receiving electroconvulsive therapy, and having vision loss substantially.

\section{Procedure}

All participants were administered SCID-I (21), and patients with OCD had also administered YaleBrown Obsessive Compulsive Scale (Y-BOCS) $(22,23)$ and Hamilton Depression Rating Scale (HAM-D) (24). Participants in the healthy control group were evaluated to ensure the absence of psychiatric diagnosis based on SCID-I. In order to assess neurocognitive flexibility, all participants were given Berg Card Sorting Test (BCST), Trail Making Test (TMT), and Category Fluency Test (CF) (25-27). Participants completed BCST via computer, whereas TMT and CF were performed by using paper and pencil. Hewitt Multidimensional Perfectionism Scale (HMPS) and Obsessive Beliefs Questionnaire-44 (OBQ-44) were also given to all participants. Three OCD subtypes were constituted as i)Contamination: related to hygiene obsessions/compulsions Unacceptable Thoughts: related to religious, sexual, and aggression. For instance, fear of hurting someone or self, doing something to be ashamed of, being sinful, intrusive sexual thoughts. iii)Checking/Symmetry/Ordering/Counting/Ritual symptoms: included symmetry of stuff, repetitive behaviors, counting and ordering goods, compulsive checking behavior. For example, patients who show contamination symptoms were coded as ' 1 ', and patients who do not show these symptoms were coded as ' 0 '. Similarly, patients diagnosed with unacceptable thoughts were coded as ' 1 ', others who does not have these symptoms were ' 0 '. Neurocognitive test performance, scores of clinical tests, and scales were evaluated between ' 1 ' and ' 0 ' for each OCD subtype. The procedure for each participant was approximately 75 minutes.

\section{Measures}

Structured Clinical Interview for the DSM-IV Axis I Disorders: It is a structured clinical interview form developed for assessing DSM-IV Axis I disorders. SCID-I provides a standard and systematic procedure for diagnostic evaluation, and it increases the reliability and validity of the diagnosis. (21) Validity and reliability studies of SCID-I were carried out (Kappa coefficient as 0.86 and diagnostic agreement 98\%) by Özkürkçügil et al. in 1999 (28).

Berg Card Sorting Test: BCST is a free version of the WCST in 'Psychology Experiment Building Language (PEBL) 2.0 software used to assess cognitive flexibility and executive functions (25). The test has 128 cards on the computer screen, and each card has a particular color (green, red, blue, and yellow), shape (triangles, circles, or crosses), and several figures $(1,2,3,4)$. Participants need to sort the deck of cards based on these rules. Participants do not know the principle of the rules. Therefore, they should figure out which sorting rule is applicable by depending on feedback (correct/incorrect) from the computer, and they should change the strategy when the rule changes. The rule changes without any warning and there are nine categories to complete. Each category has its own sorting rules. The outcome measures in this study are the number of categories completed (BCST-NC), correct response(\%)(BCST-CR), perseverative error(\%) (BCST-PE (\%)), failure to maintain set (BCST-FMS), conceptual level responses\% (BCST-CLR $(\%)$ ) (29). The validity and reliability of the PEBL software tests were studied; the test- 
retest correlations were reported as moderate $(\geq .30)$ for the BCST (30).

Trail Making Test: TMT comprises A and B forms and measures attention, psychomotor speed, and cognitive flexibility. The A form mainly assesses the focused attention, whereas the B form evaluates executive functions and visual working memory. Outcome measures were time to complete A and B form and time difference between B and A (B-A) in our study (26). The test-retest reliability coefficient was reported between 0.71-0.87 (31).

Category Fluency: CF measures language ability and executive functions (27). Participants are asked to say animal names (dog, cat, cow, and so on) in one minute as much as they can. A total number of animal names and perseverations were recorded. In addition to this, switching between animal categories may represent frontal lobe activation. Thus, the category switching method was also assessed as an outcome measure demonstrated in Troyer et al.,1997 (32). For both letter and semantic fluency, test-retest correlations were reported as above 0.70 (26). The total number of animals (CF-NA), perseveration (CF-P), switching (CF-S) scores were evaluated.

Yale-Brown Obsessive Compulsive Scale: It assesses the severity and type of OCD symptoms $(22,23)$. Turkish validity and reliability of YBOC-S were performed (33). Good interrater reliability, internal consistency, and convergent validity were estimated for Y-BOCS (34).

Hamilton Depression Rating Scale: HAM-D is a 17-item test that assesses the severity of depression in the last one week (24). Test-retest reliability is 0.85 , Cronbach's alpha reliability coefficient was reported 0.75 , Turkish validity and reliability study were performed (35).

Obsessive Beliefs Questionnaire-44: OBQ-44 is a self-report instrument that evaluates obsessional beliefs. It includes three subscales; 1)Inflated Responsibility and Threat Overestimation; 2)Perfectionism and Intolerance of Uncertainty; 3) Over-importance and Over-control of Thoughts. Good internal consistency and convergent validity were indicated for OBQ-44 (20). It was adapted 442 into Turkish (36).

Hewitt Multidimensional Perfectionism Scale (HMPS): HMPS evaluates perfectionist personality traits in adults (37). Participants need to make 7point ratings of these items. It was adapted into Turkish (38). It is a 45 items measure that consists of three subscales; (i) self-oriented, (ii) other-oriented, (iii) socially prescribed (39). The internal consistency coefficient for the total HMPS score was 0.91. Cronbach alpha values for subscales varied between 0.64 and 0.94 (40).

\section{Statistical Analysis}

In order to evaluate the differences between the two groups, the T-Test was used for the data showing the normal distribution, and the MannWhitney-U test was used for the data not showing normal distribution. Spearman rank-order correlation with Bonferroni correction tests was needed to evaluate the correlations between clinical and neurocognitive functions data. Statistical Package for Social Sciences (SPSS) for Windows 20 package program was used for statistical analysis. P-value $<0.05$ was taken as statistically significant.

\section{RESULTS}

The socio-demographic characteristics of the two sample groups and comorbid psychiatric conditions of OCD patients were summarized in Table 1. Most of the patients were taking an antidepressant (\%50) and combined medications (antidepressants, antipsychotics, and/or anxiolytics) (\%35). There were only a few patients $(15 \%)$ who were not taking medication. The mean YBOC-S, HAM-D, and HMPS scores were summarized in Table 2. YBOCS scores of patients were compared with neuropsychological outcomes. There was a significantly positive correlation between symptom severity and TMT-A completion time $(\mathrm{r}=0.305, \mathrm{p}=0.013)$. There was no statistically significant relationship between symptom severity and other NPT scores ( $p>0.05)$. (see Table 3). Mann Whitney U test was applied to evaluate differences between NPT performance of OCD sample with depression and without depression. Results showed that there was not statistically significant differences between these two samples $(\mathrm{p}>0.05)$. 
Neurocognitive flexibility, perfectionism, obsessive beliefs in patients with obsessive compulsive disorder

\begin{tabular}{|c|c|c|c|c|c|c|}
\hline & OCD & \multicolumn{2}{|l|}{ HC } & \multirow{2}{*}{$d f$} & \multirow{2}{*}{$t$} & \multirow{2}{*}{$p$} \\
\hline & $M \quad S D$ & $M$ & $S D$ & & & \\
\hline Age & $\begin{array}{ll}30.89 & 10.83\end{array}$ & 32.32 & 10.50 & 139 & -0.793 & 0.429 \\
\hline \multirow[t]{3}{*}{ Education } & $13.03 \quad 3.41$ & 13.71 & 4.11 & 139 & -1.056 & 0.293 \\
\hline & OCD n $(\%)$ & \multicolumn{2}{|c|}{$\mathrm{HC} \mathrm{n}(\%)$} & \multicolumn{3}{|c|}{ Group comparisons } \\
\hline & & & & $\chi \dagger$ & $d f$ & $p$ \\
\hline Gender & & & & 0.220 & 1 & 0.64 \\
\hline Female & $43(65.15)$ & $46(61$ & & & & \\
\hline Male & $23(34.85)$ & $29(38$ & & & & \\
\hline Marital Status & & & & 1.801 & 2 & 0.41 \\
\hline Married & $24(36.36)$ & $35(46$ & & & & \\
\hline Single & $40(60.61)$ & $37(49$ & & & & \\
\hline Divorced & $2(3.03)$ & $3(4.0)$ & & & & \\
\hline Job Status & & & & 13.797 & 1 & $0.00 *$ \\
\hline Working & 19 (28.79) & $45(60$ & & & & \\
\hline Not working & $47(71.21)$ & $30(40$ & & & & \\
\hline Comorbidities & OCD n $(\%)$ & & & & & \\
\hline MDD(recent) & $18(27.3)$ & & & & & \\
\hline MDD(remission) & $11(16.7)$ & & & & & \\
\hline Dysthymia & $17(25.8)$ & & & & & \\
\hline GAD & $16(24.2)$ & & & & & \\
\hline $\mathrm{PD}($ recent $)$ & $3(4.5)$ & & & & & \\
\hline $\mathrm{PD}$ (remission) & $2(3)$ & & & & & \\
\hline AG(recent) & $1(1.5)$ & & & & & \\
\hline AG(remission) & $3(4.5)$ & & & & & \\
\hline Specific phobia & $18(27.3)$ & & & & & \\
\hline PTSD(recent) & $1(1.5)$ & & & & & \\
\hline PTSD(remission) & $2(3)$ & & & & & \\
\hline
\end{tabular}

Note: MDD: Major Depressive Disorder, GAD: Generalized Anxiety Disorder, PD: Panic Disorder, AD: Agoraphobia

PTSD: Post-Traumatic Stress Disorder, $\mathrm{p}<0.05$.

Mann-Whitney $\mathrm{U}$ test was conducted in order to assess neuropsychological test performance differences across groups, as shown in Table 2. Patients performed worse on TMT-A, TMT-B, and TMT(BA) compared to HC. However, analyses indicated that there were no significant performance differences on CF-NA, CF-P, CF-S and BCST-NC,
BCST-PE (\%), BCST-FMS, BCST-CLR (\%) (p > $0.05)$, whereas BCST-CR $(\%)$ of OCD patients were significantly less than $\mathrm{HC}(\mathrm{p}=0.048)$ (see Table 2).

Spearman rank-order correlation with Bonferroni correction was conducted in order to assess the

Table 2. Comparisons of clinical tests/scales scores and NPT performance of participants

\begin{tabular}{|c|c|c|c|c|c|c|c|}
\hline \multirow[t]{2}{*}{ Measure } & \multicolumn{2}{|l|}{ OCD } & \multirow{2}{*}{$\begin{array}{l}\text { Control } \\
M\end{array}$} & \multirow[b]{2}{*}{$S D$} & \multirow[b]{2}{*}{$d f$} & \multirow[b]{2}{*}{$t$} & \multirow[b]{2}{*}{$p$} \\
\hline & $M$ & $S D$ & & & & & \\
\hline HAM-D & 13.30 & 7.48 & - & - & - & - & - \\
\hline YBOC-S & 24.67 & 6.87 & - & - & - & - & - \\
\hline HMPS & 192.79 & 44.04 & 165.57 & 37.16 & 139 & 3.979 & $0.00 *$ \\
\hline OBQ-R & 65.17 & 18.03 & 51.60 & 15.72 & 139 & 4.773 & $0.00 *$ \\
\hline OBQ-P & 73.01 & 21.38 & 56.88 & 17.44 & 139 & 4.932 & $0.00 *$ \\
\hline OBQ-I & 42.06 & 15.88 & 32.99 & 10.84 & 112.591 & 3.910 & $0.00 *$ \\
\hline \multirow[t]{2}{*}{ OBQ-Total } & 180.24 & 48.32 & 141.47 & 39.11 & 139 & 5.262 & $0.00 *$ \\
\hline & $M$ & $S D$ & $M$ & $S D$ & $Z$ & $U$ & $p$ \\
\hline TMT-A & 41.77 & 19.19 & 35.23 & 17.46 & -2.470 & 1877.50 & $0.014 *$ \\
\hline TMT-B & 100.02 & 49.02 & 80.03 & 48.73 & -3.034 & 1711.50 & $0.002 *$ \\
\hline TMT (B-A) & 58.23 & 38.59 & 44.80 & 40.52 & -3.047 & 1708.50 & $0.002 *$ \\
\hline CF-NA & 19.64 & 5.73 & 20.56 & 5.00 & -0.749 & 2294.00 & 0.454 \\
\hline CF-P & 0.28 & 0.52 & 0.36 & 0.63 & -0.538 & 2373.50 & 0.591 \\
\hline CF-S & 7.97 & 2.85 & 8.20 & 2.71 & -0.343 & 2392.50 & 0.732 \\
\hline BCST-NC & 4.66 & 2.95 & 5.46 & 2.99 & -1.600 & 1971.50 & 0.110 \\
\hline BCST_CR \% & 61.07 & 17.83 & 66.98 & 15.46 & -1.975 & 1882.00 & $0.048 *$ \\
\hline BCST-PE \% & 19.70 & 11.80 & 18.91 & 10.07 & -0.436 & 2239.00 & 0.663 \\
\hline BCST-FMS & 1.05 & 1.23 & 1.14 & 1.17 & -0.724 & 2180.50 & 0.469 \\
\hline BCST-CLR $(\%)$ & 48.30 & 23.52 & 55.53 & 21.68 & -1.843 & 1912.50 & 0.065 \\
\hline
\end{tabular}

Note: NPT: Neuropsychological test, HAM -D: Hamilton Depression Rating Scale, YBOC -S: Yale -Brown Obsessive Compulsive Scale; HMPS: Hewitt Multidimensional Perfectionism Scale, OBQ: Obsessive Belief Questionnaire, OBQ -R: Inflated Responsibility and Threat Overestimation, OBQ -P: Perfecti onism and Intolerance of Uncertainty, OBQ -I: Overimportance and Over -control of Thoughts; TMT: Trail Making Test; CF: Category Fluency, NA: Number of Animals, P:

Perseveration, S: Switch; BCST: Berg Card Sorting Test, NC: Number of Categories Completed, C R: Correct Response, PE: Perseverative Error, FMS: Failure to Maintain Set, CLR: Conceptual Level Responses, ${ }^{*} p<0.05$. 
Onder Uzgan B, Tetik Oktay M, Aykac C, Ermis C, Alkin T.

\begin{tabular}{|c|c|c|c|c|c|c|c|}
\hline & OBQ-R & OBQ-P & OBQ-I & OBQ-Total & HMPS & HAM-D & Y-BOCS \\
\hline TMT-A & $\begin{array}{l}r=0.075 \\
p=0.547\end{array}$ & $\begin{array}{l}r=-0.058 \\
p=0.646\end{array}$ & $\begin{array}{l}r=0.033 \\
p=0.791\end{array}$ & $\begin{array}{l}r=0.013 \\
p=0.918\end{array}$ & $\begin{array}{l}r=-0.158 \\
p=0.206\end{array}$ & $\begin{array}{l}r=0.158 \\
p=0.204\end{array}$ & $\begin{array}{l}r=0.305 \\
p=0.013\end{array}$ \\
\hline TMT-B & $\begin{array}{l}r=-0.062 \\
p=0.625\end{array}$ & $\begin{array}{l}r=-0.185 \\
p=0.141\end{array}$ & $\begin{array}{l}r=0.073 \\
p=0.563\end{array}$ & $\begin{array}{l}r=-0.062 \\
p=0.626\end{array}$ & $\begin{array}{l}r=-0.272 \\
p=0.028\end{array}$ & $\begin{array}{l}r=0.175 \\
p=0.163\end{array}$ & $\begin{array}{l}r=0.117 \\
p=0.354\end{array}$ \\
\hline TMT (B-A) & $\begin{array}{l}r=-0.074 \\
p=0.558\end{array}$ & $\begin{array}{l}r=-0.217 \\
p=0.083\end{array}$ & $\begin{array}{l}r=0.104 \\
p=0.411\end{array}$ & $\begin{array}{l}r=-0.072 \\
p=0.570\end{array}$ & $\begin{array}{l}r=-0.246 \\
p=0.048\end{array}$ & $\begin{array}{l}r=0.146 \\
p=0.247\end{array}$ & $\begin{array}{l}r=-0.047 \\
p=0.710\end{array}$ \\
\hline CF-NA & $\begin{array}{l}\mathrm{r}=-0.010 \\
\mathrm{p}=0.935\end{array}$ & $\begin{array}{l}r=0.099 \\
p=0.428\end{array}$ & $\begin{array}{l}r=-0.148 \\
p=0.237\end{array}$ & $\begin{array}{l}r=-0.013 \\
p=0.916\end{array}$ & $\begin{array}{l}r=0.150 \\
p=0.231\end{array}$ & $\begin{array}{l}r=-0.261 \\
p=0.034\end{array}$ & $\begin{array}{l}r=-0.093 \\
p=0.459\end{array}$ \\
\hline CF-P & $\begin{array}{l}r=0.098 \\
p=0.434\end{array}$ & $\begin{array}{l}r=-0.054 \\
p=0.669\end{array}$ & $\begin{array}{l}r=0.016 \\
p=0.896\end{array}$ & $\begin{array}{l}r=0.011 \\
p=0.930\end{array}$ & $\begin{array}{l}r=0.223 \\
p=0.072\end{array}$ & $\begin{array}{l}r=-0.107 \\
p=0.395\end{array}$ & $\begin{array}{l}r=-0.124 \\
p=0.321\end{array}$ \\
\hline CF-S & $\begin{array}{l}r=-0.083 \\
p=0.508\end{array}$ & $\begin{array}{l}r=-0.028 \\
p=0.826\end{array}$ & $\begin{array}{l}r=-0.205 \\
p=0.098\end{array}$ & $\begin{array}{l}r=-0.106 \\
p=0.396\end{array}$ & $\begin{array}{l}r=0.029 \\
p=0.819\end{array}$ & $\begin{array}{l}r=-0.120 \\
p=0.336\end{array}$ & $\begin{array}{l}r=-0.209 \\
p=0.093\end{array}$ \\
\hline BCST-NC & $\begin{array}{l}r=-0.028 \\
p=0.824\end{array}$ & $\begin{array}{l}r=0.017 \\
p=0.895\end{array}$ & $\begin{array}{l}r=-0.025 \\
p=0.841\end{array}$ & $\begin{array}{l}r=0.008 \\
p=0.948\end{array}$ & $\begin{array}{l}r=0.116 \\
p=0.356\end{array}$ & $\begin{array}{l}r=-0.149 \\
p=0.236\end{array}$ & $\begin{array}{l}r=-0.166 \\
p=0.186\end{array}$ \\
\hline BCST.CR \% & $\begin{array}{l}r=-0.066 \\
p=0.603\end{array}$ & $\begin{array}{l}r=-0.027 \\
p=0.831\end{array}$ & $\begin{array}{l}r=-0.068 \\
p=0.589\end{array}$ & $\begin{array}{l}r=-0.045 \\
p=0.723\end{array}$ & $\begin{array}{l}r=0.083 \\
p=0.513\end{array}$ & $\begin{array}{l}r=-0.144 \\
p=0.251\end{array}$ & $\begin{array}{l}r=-0.182 \\
p=0.146\end{array}$ \\
\hline BCST-PE \% & $\begin{array}{l}r=0.056 \\
p=0.659\end{array}$ & $\begin{array}{l}r=-0.066 \\
p=0.599\end{array}$ & $\begin{array}{l}r=0.118 \\
p=0.349\end{array}$ & $\begin{array}{l}r=-0.002 \\
p=0.989\end{array}$ & $\begin{array}{l}r=-0.014 \\
p=0.913\end{array}$ & $\begin{array}{l}r=-0.105 \\
p=0.403\end{array}$ & $\begin{array}{l}r=-0.169 \\
p=0.178\end{array}$ \\
\hline BCST-FMS & $\begin{array}{l}r=-0.04 \\
p=0.66\end{array}$ & $\begin{array}{l}r=-0.08 \\
p=0.38\end{array}$ & $\begin{array}{l}r=0.09 \\
p=0.28\end{array}$ & $\begin{array}{l}r=-0.02 \\
p=0.86\end{array}$ & $\begin{array}{l}r=-0.10 \\
p=0.24\end{array}$ & $\begin{array}{l}r=-0.22 \\
p=0.08\end{array}$ & $\begin{array}{l}r=-0.24 \\
p=0.05\end{array}$ \\
\hline $\begin{array}{l}\text { BCST-CLR } \\
(\%) \\
(\%)\end{array}$ & $\begin{array}{l}r=-0.053 \\
p=0.676\end{array}$ & $\begin{array}{l}r=-0.014 \\
p=0.910\end{array}$ & $\begin{array}{l}r=-0.037 \\
p=0.768\end{array}$ & $\begin{array}{l}r=-0.025 \\
p=0.842\end{array}$ & $\begin{array}{l}r=0.089 \\
p=0.481\end{array}$ & $\begin{array}{l}r=-0.151 \\
p=0.231\end{array}$ & $\begin{array}{l}r=-0.205 \\
p=0.102\end{array}$ \\
\hline
\end{tabular}

Note: NPT: Neuropsychological Test, HMPS: Hewitt Multidimensional Perfectionism Scale, OBQ: Obsessive Belief Questionnaire, OBQ -R: Inflated Responsibility and Threat Overestimation, OBQ -P: Perfectionism a nd Intolerance of Uncertainty, OBQ-I: Over-importance and Over-control of Thoughts; TMT: Trail Making Test; CF: Category Fluency, NA: Number of Animals, P: Perseveration, S: Switch; BCST: Berg Card Sorting Test, NC: Number of Categories Completed, CR: Correct Response, PE: Perseverative Error, FMS: Failure to Maintain Set, CLR: Conceptual Level Responses, *p<0.007.

relationship between neuropsychological test performance (NTP) and scales (HMPS and OBQ-44) as well as NTP and clinical assessments (HAM-D and Y-BOCS). The results indicated that there was no significant association between severity scales and NTP ( $p>0.007)$. There were significant positively correlation between TMT-A and YBOC-S; negative correlation between CF-NA and HAM-D score of patients $(\mathrm{r}=0.305, \mathrm{p}=0.013 ; \mathrm{r}=-0.261$, $p=0.034$, respectively). Likewise, TMT B and TMT B-A performance and HMPS score were negatively correlated $(\mathrm{r}=-0.272, \mathrm{p}=0.028 ; \mathrm{r}=-0.246, \mathrm{p}$ $=0.048$, respectively). However, significance disappeared after the Bonferroni correction $(p<0.007)$. There was no significant correlation among other variables (See Table 3).

Independent samples t-test were conducted to compare the level of obsessive beliefs and subtypes of OCD. Results showed patients with contamination symptoms differed from those OCD patients without contamination symptoms in the 'Inflated Responsibility and Threat Overestimation subtest' $(p=0.047)$. Unacceptable thought subtype showed significantly higher scores in the 'Inflated Responsibility and Threat Overestimation subtest' than patients without these symptoms $(p=0.019)$. A Mann-Whitney U test was conducted to compare performance on TMT, CF, BCST between OCD subtypes. Results indicated contamination subtype of OCD significantly completed TMT-A in a longer period $(\mathrm{p}=0.003)$. Also, this subtype significantly showed more failure to maintain set in BCST than OCD group with no contamination symptoms ( $\mathrm{p}=$ 0.016) (see Table 4, 5 and 6).

\section{DISCUSSION}

The current study's main findings were patients had more perfectionist traits and higher-level obsessive beliefs than the healthy control group. OCD patients were slower on TMT and performed less correct responses on BCST compared to control subjects. Also, analyses of subtypes of OCD indicated that neuropsychological performance and categories of obsessive beliefs differed among subtypes. However, it seemed that cognitive inflexibility was not significantly correlated with high-level obsessive beliefs and perfectionism.

In this study, possible relationships among cognitive flexibility, perfectionism, and obsessive belief in OCD were investigated. Besides, these variables were also evaluated in three established OCD subtypes, namely, (i) Contamination, ii) Unacceptable Thoughts, iii) Checking/Symmetry/ Ordering/ Counting/Ritual Symptoms). One of the strengths 
Table 4. Comparison of NPT, OBQ, HMPS scores between patients with contamination symptoms and patients with no contamination symptoms

\begin{tabular}{llllllll}
\hline Measure & $\mathbf{S 1}$ & \multicolumn{7}{c}{ Other } & & & \\
\cline { 2 - 5 } & $M$ & $S D$ & $M$ & $S D$ & $Z$ & $U$ & $p$ \\
\hline TMT A & 44.92 & 20.00 & 28.92 & 6.10 & -3.002 & 158.500 & $\mathbf{0 . 0 0 3 *}$ \\
TMT B & 105.85 & 52.79 & 76.69 & 15.33 & -1.673 & 236.000 & 0.094 \\
TMT B-A & 60.85 & 42.15 & 47.77 & 15.52 & -0.492 & 308.000 & 0.623 \\
\hline CF-NA & 19.49 & 6.12 & 20.23 & 3.94 & -0.364 & 322.000 & 0.716 \\
CF-P & 0.28 & 0.50 & 0.31 & 0.63 & -0.127 & 338.500 & 0.899 \\
CF-S & 7.75 & 3.00 & 8.85 & 1.99 & -1.445 & 255.500 & 0.148 \\
\hline BCST-NC & 4.67 & 2.94 & 4.62 & 3.12 & -0.041 & 335.500 & 0.967 \\
BCST-CR (\%) & 60.17 & 18.44 & 64.66 & 15.24 & -0.623 & 300.000 & 0.533 \\
BCST-PE(\%) & 19.96 & 12.64 & 18.67 & 7.81 & -0.082 & 333.000 & 0.935 \\
BCST-FMS & 0.88 & 1.17 & 1.69 & 1.32 & -2.415 & 199.000 & $\mathbf{0 . 0 1 6}$ \\
BCST-CLR(\%) & 47.35 & 23.94 & 52.09 & 22.22 & -0.492 & 308.000 & 0.623 \\
\hline & $M$ & $S D$ & $M$ & $S D$ & $t$ & $d f$ & $p$ \\
OBQ-R & 67.34 & 18.15 & 56.31 & 15.06 & 2.023 & 64 & $\mathbf{0 . 0 4 7 *}$ \\
OBQ-P & 75.42 & 21.95 & 63.23 & 16.10 & 1.876 & 64 & 0.065 \\
OBQ-I & 43.13 & 15.40 & 37.69 & 17.66 & 1.109 & 64 & 0.272 \\
OBQ-Total & 185.89 & 48.80 & 157.23 & 40.11 & 1.958 & 64 & 0.055 \\
\hline HMPS & 195.40 & 43.64 & 182.15 & 45.83 & 0.971 & 64 & 0.335 \\
\hline
\end{tabular}

Note: NPT: Neuropsychological test, S1(Subtype 1): Contamination Subtype, Other: Patients with no contamination symptoms; TMT: Tra il Making Test; CF: Category Fluency, NA: Number of Animals, P: Perseveration, S: Switch; BCST: Berg Card Sorting Test, NC: Number of Categories Completed, CR: Correct Response, PE: Perseverative Error, FMS: Failure to Maintain Set, CLR: Conceptual Level R esponses; OBQ: Obsessive Belief Questionnaire, OBQ -R: Inflated Responsibility and Threat Overestimation, OBQ-P: Perfectionism and Intolerance of Uncertainty, OBQ-I: Over-importance and Over-control of Thoughts; HMPS: Hewitt Multidimensional Perfectionism Scale, ${ }^{*} p<0.05$.

of our study is that it focuses on the relationship between neurocognitive assessments and a range of psychological factors in the patient group, together with healthy controls. Another strength and distinction of our study is that the comparisons of each three dimensions of OBQ-44 with neuropsychological variables were performed, three different cognitive flexibility measurements were applied and included psychiatrically healthy control group unlike other studies beliefs $(12,41)$

Evidence indicated that patients with OCD compared to healthy subjects had low scores on WCST (10). However, in our study, patients with OCD had low scores only on BCST-CR than controls. Likewise, a study revealed that the performance of patients and healthy people on WCST was not significantly different (42). Previous research also showed that unmedicated OCD patients had reduced performance on WCST than OCD patients treated with medication. Given that medication may provide improvements in cognitive flexibility and sustained attention (43), it could be concluded that our results might be affected by medication and therapy.

Previous studies have demonstrated that OCD patients with predominant contamination fears showed poorer WCST-correct response perfor- mance (44). We found impairments in contamination OCD subtype's performance at BCST-FMS. This inconsistency among studies could be related to using different versions of the same neuropsychological assessment tool. Even though BCST and WCST are quite similar, there could be some differences in performance results. The direct comparison between WCST and BCST is needed to be further addressed (45). In the current study, the performance of TMT-A was significantly poor in the contamination subtype of OCD, and there was a trend toward impaired TMT-B. Similarly, the contamination subtype had more errors and poor performance on TMT-B (46). As seen in our results, impairment in TMT-A performance was associated with OCD symptom severity. Part A of TMT specifically measures attention; therefore, slowness on TMT-A, our OCD sample may have a deterioration in attentional focus to stimulus, due to their severe symptoms of contamination as a threatening factor for them. (47).

Another study reported that there was deterioration in TMT performance in aggression and symmetry-ordering subtypes (48). In our sample, unacceptable thoughts (including religious, sexual, and aggression) subtype showed only a trend toward poorer TMT performance. These results indicate there may be cognitive inflexibility in contamina- 
Onder Uzgan B, Tetik Oktay M, Aykac C, Ermis C, Alkin T.

Table 5. Comparison of NPT, OBQ, HMPS scores between patients with unacceptable thoughts symptoms and patients with no unacceptable symptoms

\begin{tabular}{|c|c|c|c|c|c|c|c|}
\hline & \multicolumn{2}{|l|}{ S2 } & \multirow{2}{*}{$\begin{array}{l}\text { Other } \\
M\end{array}$} & \multirow[b]{2}{*}{$S D$} & \multirow[b]{2}{*}{$Z$} & \multirow[b]{2}{*}{$U$} & \multirow[b]{2}{*}{$p$} \\
\hline & $M$ & $S D$ & & & & & \\
\hline TMT A & 43.30 & 19.70 & 32.11 & 12.32 & -1.842 & 158.000 & 0.065 \\
\hline TMT B & 103.31 & 50.71 & 79.56 & 31.67 & -1.216 & 188.000 & 0.224 \\
\hline TMT B-A & 59.97 & 39.98 & 47.44 & 27.76 & -0.836 & 208.000 & 0.403 \\
\hline CF-NA & 19.56 & 5.88 & 20.11 & 4.96 & $-0,169$ & 247.500 & 0.866 \\
\hline CF-P & 0.28 & 0.53 & 0.33 & 0.50 & -0.479 & 237.000 & 0.632 \\
\hline CF-S & 7.86 & 2.93 & 8.67 & 2.30 & -0.771 & 215.500 & 0.440 \\
\hline BCST-NC & 4.70 & 3.02 & 4.44 & 2.65 & -0.220 & 240.500 & 0.826 \\
\hline BCST-CR (\%) & 60.86 & 18.43 & 62.33 & 14.32 & 0.000 & 252.000 & 1.000 \\
\hline BCST-PE(\%) & 30.37 & 12.21 & 15.54 & 8.06 & -1.027 & 198.000 & 0.305 \\
\hline BCST-FMS & 0.96 & 1.21 & 1.56 & 1.33 & -1.479 & 178.500 & 0.139 \\
\hline \multirow[t]{2}{*}{ BCST-CLR(\%) } & 48.22 & 24.00 & 48.78 & 21.52 & -0.009 & 251.500 & 0.992 \\
\hline & $M$ & $S D$ & $M$ & $S D$ & $t$ & $d f$ & $p$ \\
\hline OBQ-R & 67.21 & 17.67 & 52.22 & 15.44 & 2.401 & 64 & 0.019* \\
\hline OBQ-P & 74.51 & 21.19 & 63.56 & 21.31 & 1.440 & 64 & 0.155 \\
\hline OBQ-I & 43.05 & 16.08 & 35.78 & 13.65 & 1.284 & 64 & 0.204 \\
\hline OBQ-Total & 184.77 & 47.53 & 151.56 & 45.60 & 1.958 & 64 & 0.055 \\
\hline HMPS & 195.42 & 44.87 & 176.11 & 36.15 & 1.227 & 64 & 0.224 \\
\hline
\end{tabular}

Note: NPT: Neuropsychological Test, S2 (Subtype 2): Unacceptable Thoughts, Other: Patients with no unacceptable thoughts symptoms; TMT: Trail Making Test; CF: Category Fluency, NA: Number of Animals, P: Perseveration, S: Switch; BCST: Berg Card Sorting Test, NC: Number of Categories Completed, CR: Correct Response, PE: Perseverative Error, FMS: Failure to Maintain Set, CLR: Conceptual Level Responses; OBQ: Obsessive Belief Questionnaire, OBQ -R: Inflated Responsibility and Threat Overestimation, OBQ-P: Perfectionism and Intolerance of Uncertainty, OBQ-I: Over-importance and Over-control of Thoughts; HMPS: Hewitt Multidimensional Perfectionism Scale, ${ }^{*} p<0.05$

tion and unacceptable thought subtypes. Also, the present study showed symmetry subtype was related to poor Category Fluency-Switch performance. Some studies suggested that switching between categories may reflect neurocognitive flexibility (32). Thus, we can speculate that patients with symmetry obsessions may have impaired cognitive flexibility.

Our TMT results were consistent with previous research. Patients were slower than healthy comparisons (48). This slower performance could be explained by psychomotor slowness because most OCD patients in our study suffered from depressive comorbidity (49). However, another research showed OCD patients without medication, and comorbid psychiatric disorders had poorer performance in TMT-A, TMT-B, and TMT(B-A) than healthy comparisons. Moreover, having poor ability on TMT(B-A) suggests cognitive flexibility is poor rather than psychomotor speed (50). Rather than psychomotor speed, TMT also assesses executive functions and attention, therefore, executive dysfunctions and impairment on attention may lead to poor performance on TMT (48).

Inconsistent with our results, some researchers have demonstrated that OCD patients with a high level of obsessive beliefs did more perseverative errors, total errors, and trials to complete the first category rather than low-level beliefs. These findings argue that a high level of obsessive beliefs may have a negative impact on cognitive flexibility $(12,41)$. This discrepancy could be attributed to differences between the mean total OBQ of our OCD sample $(M=180.24)$ and other studies' sample, Mhigh-level belief $=206.10$, Mlow-level belief=127.91 (12); Mhigh belief=206.17, Mlowlevel belief $=105.80$ (41). We can assume, our OCD sample may not have enough high-level obsessive beliefs to affect cognitive flexibility. Contrary to our expectation, neuropsychological tests and HMPS were not correlated, and we could not find that it disrupts cognitive flexibility. A possible explanation may be that the Perfectionism trait is specific to obsessive compulsive personality disorder rather than obsessive compulsive disorder (37). However, we can not rule out the thorough influences of psychotropic medications on these measures. Thus, we should not ignore the possibility that perfectionism may still disrupt cognitive flexibility because people with perfectionist tendencies may not have effective coping strategies or may have difficulties in shifting among different ideas (51).

In this study, the contamination subtype showed a higher level of 'Inflated Responsibility and Threat 
Table 6. Comparison of NPT, OBQ, HMPS scores between patients with Checking/Symmetry/Ordering symptoms and patients with none of these symptoms

\begin{tabular}{llllllll} 
& \multicolumn{1}{c}{$M$} & $S D$ & $M$ & $S D$ & $Z$ & $U$ & $p$ \\
\cline { 2 - 6 } TMT A & 40.87 & 18.30 & 45.46 & 22.93 & -0.476 & 315.000 & 0.634 \\
TMT B & 96.54 & 46.95 & 113.92 & 56.47 & -0.853 & 286.000 & 0.394 \\
TMT B-A & 56.68 & 38.02 & 68.46 & 40.66 & -1.001 & 277.000 & 0.317 \\
\hline CF-NA & 20.26 & 5.56 & 17.08 & 5.95 & -1.309 & 263.500 & 0.191 \\
CF-P & 8.34 & 2.66 & 6.46 & 3.20 & $-2,013$ & 220.500 & $\mathbf{0 . 0 4 4}$ \\
CF-S & 0.34 & 0.55 & 0.08 & 0.28 & $-1,663$ & 266.000 & 0.096 \\
\hline BCST-NC & 4.68 & 3.07 & 4.58 & 2.50 & -0.085 & 313.500 & 0.932 \\
BCST-CR(\%) & 61.05 & 18.21 & 61.13 & 16.77 & -0.194 & 306.500 & 0.846 \\
BCST-PE(\%) & 18.48 & 10.54 & 25.10 & 15.65 & -1.362 & 237.500 & 0.173 \\
BCST-FMS & 1.00 & 1.26 & 1.25 & 1.14 & -0.940 & 265.500 & 0.347 \\
BCST-CLR(\%) & 48.13 & 24.37 & 49.03 & 20.22 & -0.135 & 310.000 & 0.892 \\
\hline & $M$ & $S D$ & $M$ & $S D$ & $t$ & $d f$ & $p$ \\
OBQ-R & 66.89 & 18.17 & 58.15 & 16.27 & 1.583 & 64 & 0.118 \\
OBQ-P & 75.25 & 21.55 & 63.92 & 18.78 & 1.737 & 64 & 0.087 \\
OBQ-I & 42.98 & 15.79 & 38.31 & 16.30 & 0.950 & 64 & 0.346 \\
OBQ-Total & 185.11 & 48.16 & 160.38 & 45.40 & 1.677 & 64 & 0.099 \\
\hline HMPS & 194.47 & 44.38 & 185.92 & 43.68 & 0.624 & 64 & 0.535 \\
\hline
\end{tabular}

Note: NPT: Neuropsychological Test, S3 (Subtype 3): Checking/Symmetry/Ordering/Counting/Ritual symptoms, Other: Patients with none of these symptoms; TMT: Trail Making Test; CF: C ategory Fluency, NA: Number of Animals, P: Perseveration, S: Switch; BCST: Berg Card Sorting Test, NC: Number of Categories Completed, CR: Correct Response, PE: Perseverative Error, FMS: Failure to Maintain Set, CLR: Conceptual Level Responses; OBQ: Obsessive Belief Questionnaire, OBQ -R: Inflated Responsibility and Threat Overestimation, OBQ -P: Perfectionism and Intolerance of Uncertainty, OBQ -I: Over -importance and Over -control of Thoughts, HMPS: Hewitt Multidimensional Perfectionism Scale, ${ }^{*} p<0.05$.

Overestimation subtest' and a trend toward perfectionism. Similarly, research showed that the 'Inflated Responsibility and Threat Overestimation' subtest scores predicted contamination symptoms and unacceptable thoughts were related to threat estimation and importance/control of thoughts in patients with OCD (44). However, our results partially support previous findings because 'unacceptable thought subtype' had a higher score only in the 'Inflated Responsibility and Threat Overestimation' subtest but not in the 'Over-importance and Over-control of Thoughts' subtest. Apart from these, research further demonstrated that OCD patients with symmetry obsessions were perfectionists, and these patients engaged in 'something is incomplete' thoughts (44). In our sample, the symmetry subtype showed only a trend in the perfectionism construct. It seems that with larger sample size, we might observe a significant relation.

The first limitation of this study is that the patient group was not a homogeneous sample of OCD in terms of current comorbid psychiatric disorders and psychotropic medications. Second, the number of neuropsychological tests applied for assessing cognitive flexibility may be insufficient to reveal a relationship between cognitive flexibility and perfectionism and obsessive beliefs.

\section{CONCLUSION}

We recommend further research to address neurocognitive etiology and clinical symptoms of OCD by diversifying cognitive flexibility measurements and using other relevant biological variables. For example, neuroimmunological mechanisms behind neurocognitive functions and obsessive beliefs in different OCD subtypes need to be examined in future studies (52). Last, various more subtypes of OCD may emerge by increasing the sample size. It is noteworthy to constitute symptom subtypes in OCD studies because every subtype has its unique symptoms. Each of them may show differences in neuropsychological functions and obsessive beliefs. Also, in terms of individualization of the treatment, research about OCD subtypes may provide benefits. In short, future research should focus effect of neurobiological factors on cognitive flexibility and clinical outcomes of different OCD subtypes.

*This study was presented as Poster Proceeding at 55. National Congress of Psychiatry

Correspondence address: Res. Assis. Betul Onder Uzgan, Çanakkale Onsekiz Mart University, Faculty of Arts And Science, Department of Biology, Çanakkale, Turkey betulonder.uzgan@comu.edu.tr 


\section{REFERENCES}

1. Diamond A. Executive functions. Annu Rev Psychol 2013; 64 : 135-168. 10.1146/annurev-psych-113011-143750

2. Lezak MD. The problem of assessing executive functions. Int J Psychol 1982; 17: 281-297. https://doi.org/10.1080/00207598208247445

3. Remijnse PL, Nielen MM, van Balkom AJ, Cath DC, van Oppen P, Uylings HB, Veltman DJ. Reduced orbitofrontal-striatal activity on a reversal learning task in obsessive-compulsive disorder. Arch Gen Psychiatry 2006; 63: 1225-1236. doi:10.1001/archpsyc.63.11.1225

4. Van Den Heuvel OA, Veltman DJ, Groenewegen HJ, Cath DC, Van Balkom AJ, Van Hartskamp J, Barkhof F, Van Dyck R. Frontal-striatal dysfunction during planning in obsessive-compulsive disorder. Arch Gen Psychiatry 2005; 62: 301-309. doi:10.1001/archpsyc.62.3.301.

5. Dittrich WH, Johansen T. Cognitive deficits of executive functions and decision-making in obsessive-compulsive disorder. Scand J Psychol 2013; 54: 393-400. https://doi.org/10.1111/sjop.12066

6. Döndü A, Memiş ÇÖ, Sevinçok L. Evaluation of neurocognitive deficits in obsessive compulsive disorder with Adas-cog: A comparative study with the healthy control group. Klin Psikiyatr Derg 2016; 19: 156-166.

7. Logue SF, Gould TJ. The neural and genetic basis of executive function: attention, cognitive flexibility, and response inhibition. Pharmacol Biochem Behav 2014; 123: 45-54. https://doi.org/10.1016/j.pbb.2013.08.007

8. Spiro RJ. Collins BP, Thota JJ, Feltovich PJ. Cognitive flexibility theory: Hypermedia for complex learning, adaptive knowledge application, and experience acceleration. Educ Technol 2003; 43: 5-10. Retrieved from www.jstor.org/stable/44429454.

9. Penadés R, Catalán R, Andrés S, Salamero M, Gastó C. Executive function and nonverbal memory in obsessive-compulsive disorder. Psychiatry Res 2005; 133: 81-90. https://doi.org/10.1016/j.psychres.2004.09.005

10. Okasha A, Rafaat M, Mahallawy N, Nahas GE, Dawla ASE, Sayed M, Kholi SE. Cognitive dysfunction in obsessive-compulsive disorder. Acta Psychiatr Scand 2000; 101: 281-285. https://doi.org/10.1034/j.1600-0447.2000.101004281.x

11. Chamberlain SR, Blackwell AD, Fineberg NA, Robbins TW, Sahakian BJ. The neuropsychology of obsessive compulsive disorder: the importance of failures in cognitive and behavioural inhibition as candidate endophenotypic markers. Neurosci Biobehav Rev 2005; 29: 399-419. https://doi.org/10.1016/j.neubiorev.2004.11.006

12. Bradbury C, Cassin SE, Rector NA. Obsessive beliefs and neurocognitive flexibility in obsessive-compulsive disorder. Psychiatry Res 2011; 18: 160-165. https://doi.org/10.1016/j.psychres.2010.11.008

13. Kim KL, Christensen RE, Ruggieri A, Schettini E, Freeman JB, Garcia AM, Dickstein DP. Cognitive performance of youth with primary generalized anxiety disorder versus primary obsessive-compulsive disorder. Depress Anxiety 2019; 36: 130-140. https://doi.org/10.1002/da.22848

14. Bloch MH, Landeros-Weisenberger A, Rosario MC,
Pittenger C, Leckman JF. Metaanalysis of the symptom structure of obsessive-compulsive disorder. Am J Psychiatry 2008; 165: 1532-1542. https://doi.org/10.1176/appi.ajp.2008.08020320

15. Leckman JF, Grice DE, Boardman J, Zhang H, Vitale A, Bondi C, Goodman W. Symptoms of obsessive-compulsive disorder. Am J Psychiatry 1997; 154: 911-917.

16. Mataix-Cols D, Marks IM, Greist JH, Kobak KA, Baer L. Obsessive-compulsive symptom dimensions as predictors of compliance with and response to behaviour therapy: results from a controlled trial. Psychother Psychosom 2002; 71: 255-262.

17. Bragdon LB, Gibb BE, Coles ME. Does neuropsychological performance in OCD relate to different symptoms? A meta-analysis comparing the symmetry and obsessing dimensions. Depress Anxiety 2018; 35: 761-774. https://doi.org/10.1002/da.22785

18. Nakao T, Okada K, Kanba S. Neurobiological model of obsessive-compulsive disorder: evidence from recent neuropsychological and neuroimaging findings. Psychiatry Clin Neurosci 2014; 68: 587-605.

19. Mataix-Cols D, Wooderson S, Lawrence N, Brammer MJ, Speckens A, Phillips ML. Distinct neural correlates of washing, checking, and hoarding symptomdimensions in obsessive-compulsive disorder. Arch Gen Psychiatry 2004; 61: 564-576.

20. Obsessive Compulsive Cognitions Working Group. Psychometric validation of the Obsessive Belief Questionnaire and interpretation of intrusions inventory-Part 2: Factor analyses and testing of a brief version. Behav Res Ther 2005; 43: 1527-1542. https://doi.org/10.1016/j.brat.2004.07.010 (Degi isminden sonraki virgül silindi)

21. First MB, Spitzer RL, Gibbon M, Williams JB. User's guide for the Structured clinical interview for DSM-IV axis I disorders SCID-I: clinician version: American Psychiatric Pub, 1997. (Dergi isminden sonraki nokta silinip virgül yapıldı)

22. Goodman WK, Price LH, Rasmussen SA, Mazure C, Fleischmann RL, Hill CL, Charney DS. The Yale-Brown Obsessive Compulsive Scale: I. Development, use, and reliability. Arch Gen Psychiatry 1989a; 46: 1006-1011. doi:10.1001/archpsyc.1989.01810110048007

23. Goodman WK, Price LH, Rasmussen SA, Mazure C, Delgado P, Heninger GR, Charney DS. The Yale-Brown Obsessive Compulsive Scale: II. Validity. Arch Gen Psychiatry 1989b; 46: 1012-1016. doi:10.1001/archpsyc.1989.01810110054008

24. Hamilton M. A rating scale for depression. J Neurol Neurosurg Psychiatry 1960; 23: 56-62. http://dx.doi.org/10.1136/jnnp.23.1.56

25. Mueller ST, Piper BJ. The psychology experiment building language (PEBL) and PEBL test battery. J Neurosci Methods 2014; 222: 250-259. https://doi.org/10.1016/j.jneumeth.2013.10.024

26. Spreen O, Strauss EA. Compendium of neuropsychological tests: Administration, norms, and commentary. New York, NY: Oxford University Press, 1998.

27. Tumaç A. Normal Deneklerde, Frontal Hasarlara Duyarlı Bazı Testlerde Performansa Yaş ve Eğitimin Etkisi. İstanbul 
Üniversitesi Sosyal Bilimler Enstitüsü, Yüksek Lisans Tezi. 1997.

28. Özkürkçügil A, Aydemir Ö, Yıldız M, Esen-Danaci A, Köroğlu E. Adaptation into Turkish and reliability of structured clinical interview for DSM-IV (SCID). İlaç Tedavi Derg 1999; 12: $233-236$

29. Fox CJ, Mueller ST, Gray HM, Raber J, Piper BJ. Evaluation of a short-form of the Berg Card Sorting Test. PloS ONE 2013;8:e63885. https://doi.org/10.1371/journal.pone.0063885.

30. Piper BJ, Mueller ST, Geerken AR, Dixon KL, Kroliczak G, Olsen RH, Miller JK. Reliability and validity of neurobehavioral function on the psychology experimental building language test battery in young adults. Peer J 2015; 3: e1460. https://doi.org/10.7717/peerj.1460

31. Türkeş N, Can H, Kurt M, Dikeç BE. İz Sürme Testi'nin 2049 yaş aralığında Türkiye için norm belirleme çalışması. Turk Psikiyatri Derg 2015; 26: 189-196. Retrieved from https://www.proquest.com/scholarly-journals/study-determinenorms-trail-making-test-age-range/docview/1739159696/se2 ? accountid $=15572$.

32. Troyer AK, Moscovitch M, Winocur G. Clustering and switching as two components of verbal fluency: Evidence from younger and older healthy adults. Neuropsychology 1997; 11: 138-146 https://doi.org/10.1037/0894-4105.11.1.138

33. Karamustafalığlu O, Üçışı A, Ulusoy M, Erkmen H. YaleBrown obsesyon kompulsiyon derecelendirme ölçeğinin geçerlik ve güvenirlik çalışması (Validity and reliability study of yaleBrown obsession compulsion rating scale: In Turkish population. 29. Ulusal Psikiyatri Kongresi 1993.

34. Taylor S. Assessment of obsessions and compulsions: Reliability, validity, and sensitivity to treatment effects. Clin Psychol Rev 1995; 15: 261-296.

35. Akdemir A, Türkçapar MH, Örsel SD, Demirergi N, Dağ I, Özbay MH. Reliability and validity of the Turkish version of the Hamilton Depression Rating Scale. Compr Psychiatry 2001; 42: 161-165. https://doi.org/10.1053/comp.2001.19756

36. Boysan M, Beşiroğlu L, Cetinkaya N, Atlı A, Aydın A. The validity and reliability of the Turkish version of the obsessive beliefs questionnaire-44 (OBQ-44)/Obsesif inanislar olcegi44'un (OIO-44) Turkce formunun Gecerlik ve Guvenirliligi. Noro Psikiyatr Ars 2010; 47: 216-222.

37. Hewitt PL, Flett GL. Perfectionism in the self and social contexts: conceptualization, assessment, and association with psychopathology. J Pers Soc Psychol 1991; 60: 456-473 https://doi.org/10.1037/0022-3514.60.3.456

38. Oral M. Üniversite Öğrencilerinde Mükemmelliyetçi Kişilik Özelliği ve Yaşam Olaylarının Depresyon Belirtileriyle İlişkisi 'Depresyona Yatkınlık-Stress Modelinin İncelenmesi. Orta Doğu Teknik Üniversitesi Sosyal Bilimler Üniversitesi, Yüksek Lisans Tezi. 1999

39. Kiral E. The Relationship between Locus of Control and Perfectionism Perception of the Primary School Administrators. Procedia Soc Behav Sci 2015; 174: 3893-3902. https://doi.org/10.1016/j.sbspro.2015.01.1130.

40. Kagan M. Frost Çok Boyutlu Mükemmelliyetçilik Ölçeginin Türkçe formunun psikometrik özellikleri/Psychometric proper- ties of the Turkish version of the Frost Multidimensional Perfectionism Scale. Anadolu Psikiyatr Derg 2011; 12: 192-197.

41. Şahin H, Köşger F, Eşsizoğlu A, Aksaray G. The Relationship Between Obsessive Belief Level and Cognitive Flexibility in Patients with Obsessive Compulsive Disorder. Noro Psikiyatr Ars 2018; 55: 376-379.

42. Gross-Isseroff R, Sasson Y, Voet H, Hendler T, LucaHaimovici K, Kandel-Sussman H, Zohar J. Alternation learning in obsessive-compulsive disorder. Biol Psychiatry 1996; 39: 733738. https://doi.org/10.1016/0006-3223(95)00179-4

43. Abbruzzese M, Ferri S, Scarone S. Wisconsin Card Sorting Test performance in obsessive compulsive disorder: no evidence for involvement of dorsolateral prefrontal cortex. Psychiatry Res 1995; 58: 37-43. https://doi.org/10.1016/0165-1781(95)02670-R

44. Pedron AC, Ferrão YA, Gurgel LG, Reppold CT. Relations between executive functions and different symptomatic dimensions in obsessive compulsive disorder. Paidéia 2015; 25: 229239. http://dx.doi.org/10.1590/1982-43272561201511

45. Piper BJ, Li V, Eiwaz MA, Kobel YV, Benice TS, Chu AM, Mueller ST. Executive function on the psychology experiment building language tests. Behav Res Methods 2012; 44: 110-123. https://doi.org/10.3758/s13428-011-0096-6

46. Durán ET, Garcell JR, Zamorano ER, Mendoza CL. Neuropsychological characterization in clinical subtypes of an obsessive-compulsive disorder (OCD) sample of patients. Salud Mental 2007; 30: 1-8.

47. Oosterman JM, Vogels RL, van Harten B, Gouw AA, Poggesi A, Scheltens P, Kessels RPC, Scherder EJ. Assessing mental flexibility: neuroanatomical and neuropsychological correlates of the Trail Making Test in elderly people. Clin Neuropsychol 2010; 24:203-219.

48. Hashimoto N, Nakaaki S, Omori IM, Fujioi J, Noguchi Y, Murata Y, Furukawa TA. Distinct neuropsychological profiles of three major symptom dimensions in obsessive-compulsive disorder. Psychiatry Res 2011; 187: 166-173. https://doi.org/10.1016/j.psychres.2010.08.001

49. Sobin C, Sackeim HA. Psychomotor symptoms of depression. Am J Psychiatry 1997; 154: 4-17. https://doi.org/10.1176/ajp.154.1.4

50. Tükel R, Gürvit H, Aslantaş Ertekin A, Oflaz S, Ertekin E, Baran B, Akça Kalem S, Kandemir PE, Alyanak Özdemiroğlu F, Atalay F. Neuropsychological function in obsessive-compulsive disorder. Compr Psychiatry 2012; 53: 167-175. https://doi.org/10.1016/j.comppsych.2011.03.007

51. Dunkley DM, Blankstein KR. Self-critical perfectionism, coping, hassles, and current distress: A structural equation modeling approach. Cogn Ther Res 2000; 24: 713-730. https://doi.org/10.1023/A:1005543529245

52. Karagüzel EÖ, Arslan FC, Uysal EK, Demir S, Aykut DS, Tat M, Karahan SC. Blood levels of interleukin-1 beta, interleukin- 6 and tumor necrosis factor-alpha and cognitive functions in patients with obsessive compulsive disorder. Compr Psychiatry 2019;89: 61-66. 